\title{
EGF stimulates cyclooxygenase-2 expression through the STAT5 signaling pathway in human lung adenocarcinoma A549 cells
}

\author{
SHOUQIANG CAO ${ }^{1}$, YUBO YAN ${ }^{1}$, XIANGYU ZHANG $^{1}, \mathrm{KAI} \mathrm{ZHANG}^{1}, \mathrm{CHENG} \mathrm{LIU}^{1}$, GUIBIN ZHAO ${ }^{1}$,

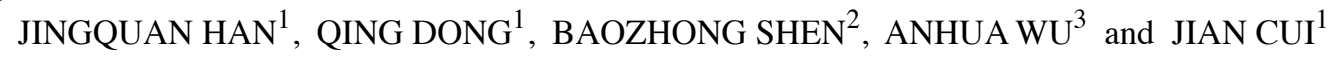

\begin{abstract}
Departments of ${ }^{1}$ Thoracic Surgery and ${ }^{2}$ Radiology, The Fourth Affiliated Hospital of Harbin Medical University, Harbin; ${ }^{3}$ Department of Neurosurgery, The First Affiliated Hospital of China Medical University, Shenyang, P.R. China
\end{abstract}

Received February 23, 2011; Accepted April 19, 2011

DOI: $10.3892 /$ ijo. 2011.1053

\begin{abstract}
The epidermal growth factor receptor (EGFR) can be activated by several growth factors within the tumor microenvironment, and it can activate several signaling pathways. For tumor development, these EGFR-related signaling pathways may converge on several common nuclear transcription factors, one such transcription factor being STAT5. STAT5 plays an important role in the oncogenic signal transduction pathway in non-small cell lung cancer. In this study, we examined whether the epidermal growth factor (EGF) can stimulate cyclooxygenase-2 (COX-2) expression in human lung adenocarcinoma A549 cells transfected with or without STAT5 siRNA or dominant-negative (DN)-STAT5, and identified the pathways involved in this response. We found that STAT5 siRNA significantly reduced EGF-induced COX-2 expression, and STAT5 phosphorylation. STAT5 phosphorylation predominantly mediates EGF-induced COX-2 promoter activity. STAT5 siRNA was found to inhibit COX-2 expression in resting A549 cells despite the absence of detectable activated phosphorylated STAT5. Using an adenoviral system, we expressed DN-STAT5 in human lung adenocarcinoma A549 cells in order to broaden the investigation and to determine the role of STAT5 in EGF-mediated COX-2 gene expression. The overexpression of DN-STAT5 significantly inhibited EGF-induced COX-2 expression, and we found that EGF induced the tyrosine phosphorylation of STAT5 and upregulated COX-2 expression. DN-STAT5 also blocked COX-2 promoter activity. Our results demonstrate that EGF stimulates COX-2 expression in human lung adenocarcinoma A549 cells via the activation of the STAT5 pathway and that COX-2
\end{abstract}

Correspondence to: Professor Jian Cui, Department of Thoracic Surgery, The Fourth Affiliated Hospital of Harbin Medical University, No. 37 Yi yuan Street, Nangang District, Harbin, Heilongjiang Province 150001, P.R. China

E-mail: mdjiancui@hotmail.com

Key words: STAT5, lung adenocarcinoma, A549, epidermal growth factor, cyclooxygenase-2 expression may be independent of phosphorylated STAT5 in A549 cells in vitro.

\section{Introduction}

Lung cancer is the number one cause of cancer related death in the world. Non-small cell lung cancer (NSCLC) is the most common lung malignant tumor. Human lung adenocarcinoma is a type of NSCLC, and despite the combined treatment strategy, the clinical outcome for patients with lung adenocarcinoma is still poor. In NSCLC, one of the major pathways that promote cellular survival and invasion is the epidermal growth factor receptor (EGFR) pathway. EGFR is a member of the ErbB family of tyrosine kinase receptors. These membrane proteins are activated by extracellular ligands of the epidermal growth factor (EGF) family, resulting in a cascade of cytoplasmic signaling events $(1,2)$. EGF receptor signalling can promote tumorigenesis by increasing cell proliferation, tissue invasion, angiogenesis and tumor cell chemoresistance (3). Human lung adenocarcinoma A549 cells contain wild-type EGFR. The activation of EGFR has been demonstrated to be correlated with the development and poor clinical outcomes of lung adenocarcinoma (4). The overexpression and autocrine activation of the normal EGF receptor plays an important role in human carcinomas.

STAT5 belongs to the STAT family, which represents a family of transcription factors that are located in the cytoplasm and are activated by a variety of different stimuli, such as cytokines and growth hormones. STAT proteins are involved in a wide variety of cellular processes, including differentiation, survival, or cell growth. STATs remain latent in the cytoplasm, and after the binding of a cytokine or growth factor to their receptors, they phosphorylate, dimerize and translocate to the nucleus, where they bind with DNA and initiate gene expression. Alterations in their activation have been demonstrated in various diseases. STAT5 is expressed in most tissues, and the constitutive activation of STAT5 has been shown to be involved in the malignant transformation of hematological malignancies, as well as breast and prostate cancer (5-7). There have been very few data on the role of STAT5 in NSCLC cells, as well as its activation status.

Prostaglandin-endoperoxide synthase (PTGS), also known as cyclooxygenase (COX), is the key enzyme in the biosynthesis 
of the prostanoids. COX is comprised of three categories, including COX-1, COX-2 and COX-3 (8). COX-1 is constitutively expressed in many cell types (9), while COX-2 is not avidly expressed under normal conditions, but it is induced in response to several stimuli (10). COX-3 is a newly discovered, paracetamol-inhibited, $\mathrm{COX}$ isoform that appears to be a splicing variant of COX-1 (11). COX-2 converts arachidonic acid to bioactive lipids including prostaglandin E2, which was found to be elevated in various types of tumors. Studies have documented that the localized expression of COX-2 and its catalyzed product, prostaglandin E2, are sufficient in initiating and progressing tumors in situ. However, the mechanism behind the malicious activity of COX-2 remains unclear in lung adenocarcinoma. COX-2 is induced in inflammation and neoplasia by EGF, TGF $\beta$, TNF $\alpha$, hypoxia and UVB light (12-16) and is inhibited by the NSAIDs, cetuximab and celecoxib $(17,18)$. Among the various regulators of COX-2 expression, p38, ERK1/2 (19), nuclear factor- $\kappa$ B and the activated protein-1 (20) pathways are well-known upstream mediators of COX-2 in inflammation and carcinogenesis (21-23). However, it is not known whether STAT5 can modulate COX-2 expression in lung adenocarcinoma.

With these issues in mind, the aim of our study was to investigate the activation status of STAT5, and explore the function of phosphorylated (p)-STAT5 in regulating COX-2 expression of A549 cells in vitro. Our results demonstrate that EGF can directly activate p-STAT5 expression in A549 cells. We also present novel evidence that EGF activates the STAT5 pathway and that EGF exerts mediating effects on COX-2 expression through the activation of STAT5. These findings contribute to our understanding of the role of STAT5 in mediating the transcriptional activation of COX-2 by EGF, and may ultimately improve the therapy of lung cancer, including human lung adenocarcinoma.

\section{Material and methods}

Cell culture. The human A549 cell line was obtained from The Cell Bank of Type Culture Collection of Chinese Academy of Sciences (CBTCCCAS, Shanghai, China). The cells were cultured as monolayers in RPMI-1640 (Gibco, CA, USA) supplemented with $10 \%$ heat-inactivated fetal bovine serum (FBS) (Gibco), $100 \mathrm{mg} / \mathrm{l}$ streptomycin and $100 \mathrm{U} / \mathrm{ml}$ penicillin at $37^{\circ} \mathrm{C}$ in a humidified atmosphere of $95 \%$ air and $5 \% \mathrm{CO}_{2}$. The cells were plated in 12-well plates for the activation of EGF (Invitrogen, CA, USA, $100 \mathrm{ng} / \mathrm{ml}$ ) and transfection studies.

Immunofluorescence analysis. A549 cells were grown on Poly-D-lysine-coated, eight-chamber slides with 6000 cells/ chamber. After $24 \mathrm{~h}$, the cells were washed with phosphatebuffered saline (PBS) and serum-starved overnight. Duplicate chambers were stimulated with EGF, and $1 \mathrm{~h}$ after the stimulation, the cells were washed with serum-free RPMI1640, and fixed for 10 min with $4 \%$ paraformaldehyde in PBS, permeabilized with $0.25 \%$ Triton X-100 in PBS and blocked for $1 \mathrm{~h}$ in PBS supplemented with 5\% FBS. Cells were incubated overnight at $4^{\circ} \mathrm{C}$ with rabbit anti-p-STAT5a/b (Santa Cruz Biotechnology, Inc., CA, USA; 1:100 dilution) antibody, followed by washing and incubating with FITC- labeled anti-rabbit secondary antibody (Sigma, St. Louis, MO, USA; 1:200 dilution) for $2 \mathrm{~h}$. In the control samples, A549 cells were treated with an equal volume of RPMI-1640. Nuclei were counterstained with Hoechst 33258. The results were observed and photographed under a fluorescence microscope (Leica Microsystems, Germany).

RNA interference. siRNA oligos (ON-TARGETplus SMARTpool siRNA) for the knockdown of endogenous STAT5 proteins were provided by Dharmacon (Dharmacon RNA Technologies, Lafayette, CO, USA). Cells were transfected with STAT5 siRNA (100 $\mathrm{nM}$ ) by using the DharmaFECT siRNA transfection reagent (Dharmacon) according to the manufacturer's instructions. ON-TARGETplus non-targeting siRNA (Dharmacon) was used as the negative control (control siRNA), and the selective silencing of STAT5 was confirmed by Western blot analysis.

Adenovirus transfection. cDNA of a carboxyl-truncated STAT5a variant, Stat5a $\triangle 740$, which displays a dominantnegative (DN) effect on both Stat5a and Stat5b-mediated transcription, was used to block the EGF-induced STAT5 signaling (24). The adenovirus containing the DN-STAT5 (Ad-CMV5Stat5a $\triangle 740$ ) plasmid, wild-type (WT)-STAT5 (AdWT-STAT5) and the control adenovirus (control) were provided by Dr Xiaofei Qin (China Medical University). Infection with recombinant adenovirus was accomplished by exposing cells to the virus in a serum-free RPMI-1640 medium for $1.5 \mathrm{~h}$ followed by the addition of a medium with or without $10 \%$ FBS. For immunoblotting and DNA banding assay, $1 \times 10^{6}$ cells were plated in $100-\mathrm{mm}$ dishes and infected with adenovirus as indicated. To monitor adenovirus infectivity, cells were exposed to Ad-CMV5Stat5a $\triangle 740$ or AdWT-STAT5 and incubated for $24 \mathrm{~h}$.

Transcription factor binding assay. A549 cells were first transfected with Stat5a $\triangle 740$ or STAT5siRNA, and then $24 \mathrm{~h}$ later, cells were stimulated with EGF $(100 \mathrm{ng} / \mathrm{ml})$ for $1 \mathrm{~h}$. At an equal pace, A549 cells were treated with EGF or WT-STAT5, nuclear proteins were isolated using a nuclear protein isolation kit (ProteoJET ${ }^{\mathrm{TM}}$ Cytoplasmic and Nuclear Protein Extraction Kit; Fermentas, Canada) according to the manufacturer's instructions. Protein concentrations were determined using the Bradford Protein Assay Kit (Pierce, Pittsburgh, PA, USA). Biotin-labeled (and unlabeled) double-stranded probes containing the consensus binding site for STAT5 (5'-biotinAGATTTCTAGGAATTCGCAG-3') were supplied by Takara Biotechnology (Dalian, China). The assay was performed as previously described (25) except that the bound proteins were incubated with rabbit anti-p-STAT5 antibody (Santa Cruz Biotechnology; $100 \mu 1 / 1: 100)$. HRP-labeled anti-rabbit antibody (Chemicon International, Temecula, CA, USA; $100 \mu \mathrm{l} / 1: 200)$ was used as the secondary antibody.

Cellular protein preparation and Western blot analysis. A549 cells were treated with WT-STAT5, Stat5a $\triangle 740$, STAT5siRNA and/or EGF as described above. Nuclear cell protein was extracted with the ProteoJET ${ }^{\mathrm{TM}}$ Cytoplasmic and Nuclear Protein Extraction Kit (Fermentas) according to the manufacturer's instructions. Total cell protein was extracted with 
the Total Cell Protein Extraction Kit (Millipore, Billerica, MA, USA). Protein concentrations were determined using the Coomassie protein assay (Bradford). An equivalent amount of protein extract from each sample was electrophoresed by $8 \%$ SDS-PAGE and transferred to nitrocellulose membrane. Membranes were blocked for $30 \mathrm{~min}$ in $5 \%$ non-fat dried milk in PBS $/ 0.1 \%$ Tween-20, and incubated overnight at $4^{\circ} \mathrm{C}$ with the rabbit anti-human STAT5 or the p-STAT5 primary antibody (Santa Cruz Biotechnology). Rabbit anti $\beta$-actin antibody (Santa Cruz Biotechnology) was used as the normalized control for total cell extractions, and rabbit anti-Histone $\mathrm{H} 1$ antibody (Santa Cruz Biotechnology) was used as the normalized control for the nuclear fraction. The membranes were then washed three times with PBS/0.1\% Tween-20 (5 min each) and incubated with the corresponsing secondary antibodies (horseradish peroxidase-conjugated, goat antibodies to rabbit and goat antibodies to mouse) (Santa cruz Biotechnology; 1:5000) for $2 \mathrm{~h}$ at room temperature. After washing three times in PBS/0.1\% Tween-20, the membranes were developed by using the ECL detection reagents and quantified using the Sigma-Gel software (Jandel Scientific Software, Sari Kafael, CA, USA).

COX-2 promoter luciferase assays. A 2004-bp-long COX-2 promoter region spanning -2069 to -66 bp up-stream of the translational start site was cloned by PCR and subcloned into the pGL3 vector (pGL3-Cox-2). The sequence was confirmed by DNA sequencing analysis. A549 cells were seeded in 12 -well plates $\left(2 \times 10^{6}\right.$ cells/plate $)$ overnight and transiently co-transfected with pGL3-Cox-2 or the internal control, pRL-TK (internal Ctrl) (Promega Corp., Madison, WI, USA), along with indicated plasmids using Lipofectamine 2000 transfection reagent (Invitrogen), following the manufacturer's instructions. Transfected cells were serum-starved for $12 \mathrm{~h}$, followed by EGF exposure for $1 \mathrm{~h}$. Firefly and Renilla luciferase activities in cell extracts were measured using a dual-luciferase reporter assay system (Promega). The relative luciferase activity was then calculated by normalizing COX-2 promoter luciferase activity to control Renilla luciferase activity. The results were expressed as the percentage of relative luciferase activity of the control group without EGF stimulation, which was set to 1 .

RNA extraction and semi-quantitative RT-PCR assay. A549 cells were transfected with siRNA or DN-STAT5 and recovered for $24 \mathrm{~h}$ (for the control group, PBS instead of siRNAs or DN-STAT5 was applied). EGF or an equal volume of PBS was then added with fresh complete medium. At the same time, A549 cells were treated with EGF or WT-STAT5. Total RNA was extracted from A549 cells using TRIzol reagent (Invitrogen). RNA (500 ng) was used as the template for cDNA synthesis and cDNA was synthesized with the FirstStrand Synthesis System for RT-PCR (Invitrogen). RT-PCR analyses were performed using the following primer sets: COX-2 (forward, 5'-TTCAAATGAGATTGTGGGAAAATT GCT- 3'; reverse, 5' -AGATCATCTCTGCCTGAGTATCT T-3'). $\beta$-actin (forward, 5'-AAATCGTGCGTGACATTAA-3'; reverse, 5'-CTCGTCATACTCCTGCTTG-3') was used as the housekeeping gene internal control. Reactions were carried out in a Gradient Thermal Cycler (Biometra, Goettingen,
Germany) for 25 cycles which consisted of $94^{\circ} \mathrm{C} 30 \mathrm{sec}, 55^{\circ} \mathrm{C}$ $30 \mathrm{sec}, 72^{\circ} \mathrm{C} 30 \mathrm{sec}$. The reaction took place in a total volume of $25 \mu \mathrm{l}$. Products were analyzed by $2 \%$ agarose gel electrophoresis and visualized by ethidium bromide staining under ultraviolet light. All primers were synthesized by Takara Biotechnology. Results were quantified using the Sigma-Gel software (Jandel Scientific Software).

Statistical analysis. Statistical comparisons were carried out using the Student's t-test. Systat statistical software (Systat Software, San Jose, CA) was used for analysis. A value of $\mathrm{P}<0.05$ was considered to indicate statistical significance.

\section{Results}

EGF induces STAT5 activation in A549 human lung adenocarcinoma cells. To determine whether STAT5 was activated in human lung adenocarcinoma cell lines in vitro and whether EGF could increase the activation of STAT5, we performed immunofluorescence staining with anti-p-STAT5 antibody. In the untreated A549 cell line, the p-STAT5 level was mainly located in the cytoplasm (at a very low level) and did not show any staining in the nucleus. EGF stimulation significantly increased the p-STAT5 staining and induced the accumulation of p-STAT5 in the cell nuclei (Fig. 1). In order to determine whether EGF induces STAT5 phosphorylation, lung adenocarcinoma cells were lysated. Cell nuclear lysates were analyzed by SDS-PAGE followed by Western blot analysis with phospho-specific antibody against pTyr694/699 of STAT5. The treatment of cells with EGF induced a marked increase in STAT5 phosphorylation, whereas under nonstimulated conditions nuclear p-STAT5 was not detected (Fig. 2).

STAT5 siRNA inhibits both resting and EGF-induced COX-2 expression. Otherwise, WT-STAT5 increases COX-2 expression. Treatment with EGF $(100 \mathrm{ng} / \mathrm{ml})$ induced COX-2 overexpression in the A549 cells compared to the control in serum-supplemented conditions. After transfection with STAT5 siRNA but not control siRNA, the COX-2 expression of both resting and EGF-treated A549 cells was significantly inhibited (Fig. 3B), showing that STAT5 expression is a key determinant of COX-2 expression in lung adenocarcinoma cells. STAT5 siRNA was found to inhibit COX-2 expression of resting A549 cells despite the absence of detectable activated p-STAT5 (Fig. 3B). This observation suggests that STAT5 is required for COX-2 expression but may mediate its effects through pathways that are independent of phosphorylation and transcriptional activation. The selective silencing of STAT5 was confirmed by Western blot analysis (Fig. 3A), demonstrating that STAT5 RNA silencing reduced STAT5 expression.

To further elucidate this hypothesis, we then transfected A549 cells with WT-STAT5. WT-STAT5 has previously been used to selectively increase STAT5 gene expression, confirmed by Western blot analysis in our study (Fig. 5A). WT-STAT5 treatment was found to increase COX-2 RNA expression (Fig. 5C). Taken together, these findings suggest that unphosphorylated STAT5 is functional and can regulate the expression of COX-2 in A549 cells. We also found that EGF 

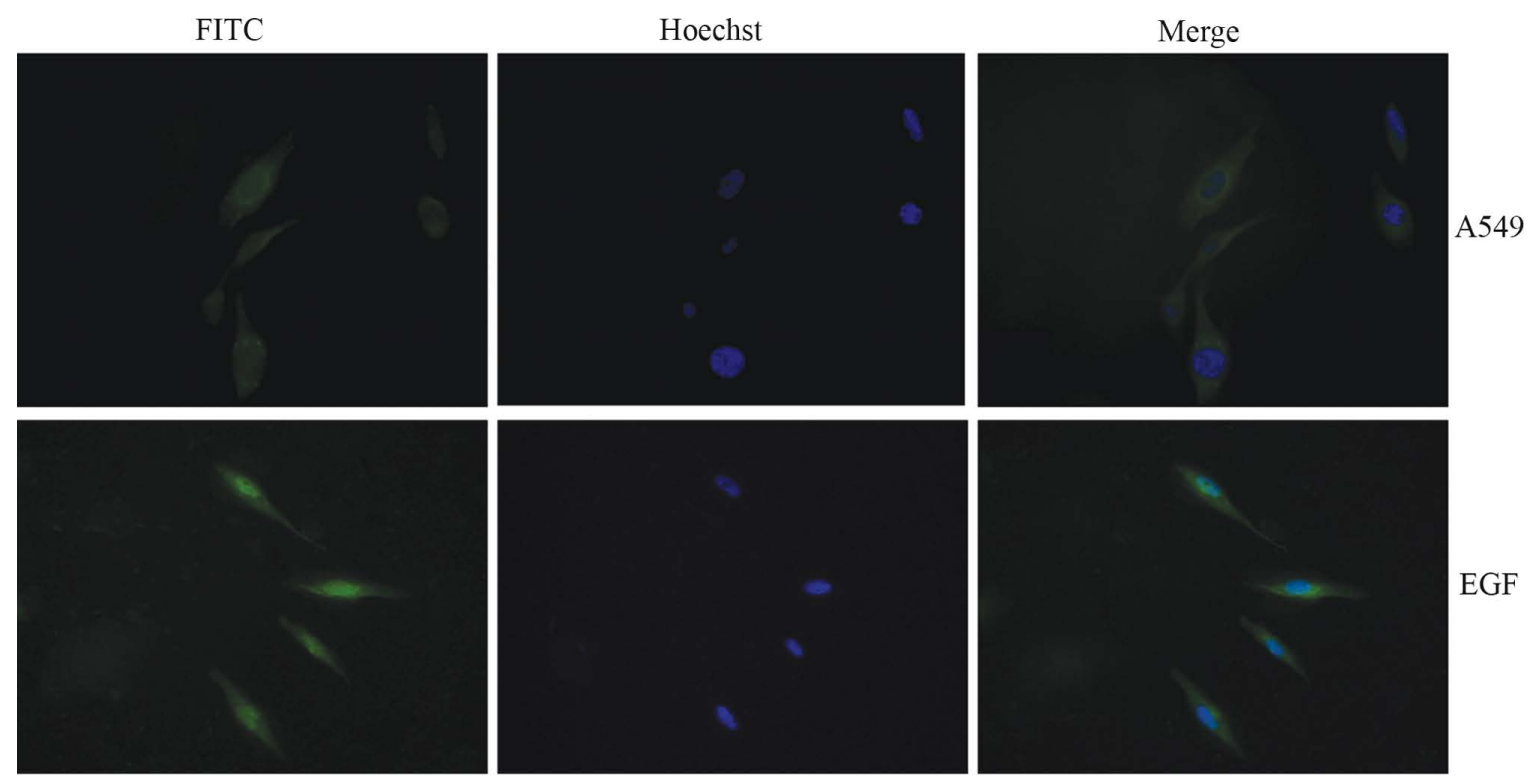

Figure 1. EGF induces STAT5 activation in human lung adenocarcinoma A549 cells. Immunofluorescence of p-STAT5 in resting and EGF-stimulated human lung adenocarcinoma A549 cells. Upper panels, no EGF stimulation; lower panels, EGF stimulation. (left panels) p-STAT5 staining (middle panels), Hoechst 33258 staining of nuclei (right panels) merged images.
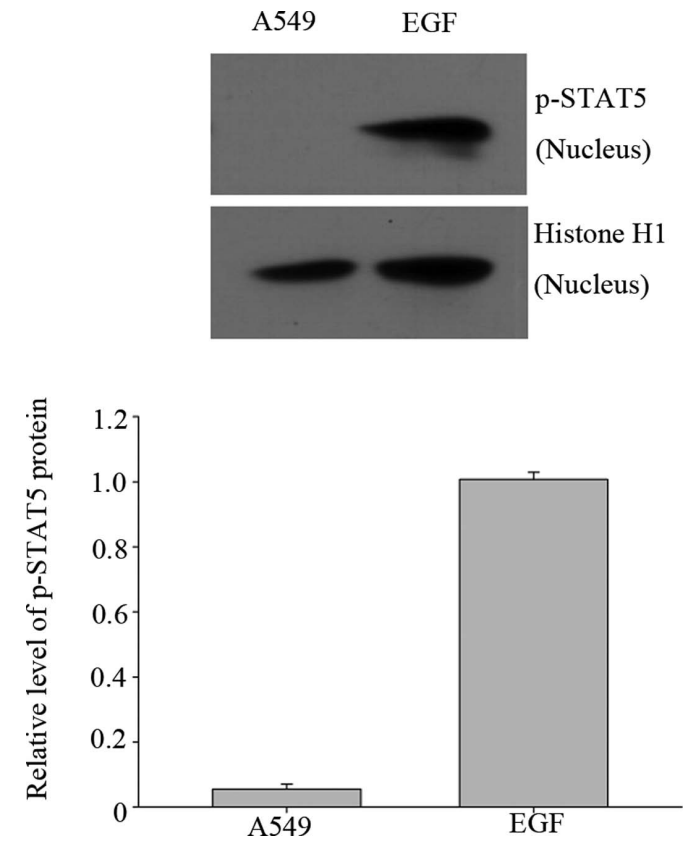

Figure 2. EGF induces p-STAT5 expression in human lung adenocarcinoma A549 cells. Upper panel, Western blot analysis of nuclear extracts from EGFstimulated and resting A549 human lung adenocarcinoma cells showing upregulation of nuclear p-STAT5. Lower panel, quantification of p-STAT5 protein levels.

can induce STAT5 activation. However, it cannot increase STAT5 expression.

STAT5 RNAi inhibits EGF-induced COX-2 promoter activity. To confirm the roles of STAT5 in COX-2 expression, we also examined the effect of STAT5 silencing by the siRNA approach in EGF-induced COX-2 promoter activity. The transfection of siRNAs targeting STAT5 significantly inhibited EGF-induced COX-2 promoter activity. However, the control siRNA did not influence EGF-mediated COX-2 promoter activity (Fig. 4). The siRNA inhibitory efficiencies were validated by Western blot analyses (Fig. 3A), demonstrating that STAT5 RNAi reduces STAT5 expression and diminishes EGF-induced STAT5 phosphorylation. Densitometric analysis of the results from Fig. 3A showed that compared to the control siRNA, STAT5 siRNA significantly inhibited EGF-induced phosphorylated and unphosphorylated STAT5 expression. Taken together, these results strongly suggest that STAT5 is an important mediator of EGF-mediated COX-2 activity.

EGF but not WT-STAT5 increases DNA binding activity of STAT5 in A549 cells. EGF stimulation was found to significantly increase the level of STAT5 phosphorylation, whereas p-STAT5 was not detected under non-stimulated and WT-STAT5 treatment conditions (Fig. 5B). These results were confirmed by Western blot analysis (Fig. 5A).

To confirm the activation of STAT5 DNA binding activity, we stimulated A549 cells with EGF or WT-STAT5. Nuclear extracts were then examined for STAT5 DNA binding activity using a biotin-labeled STAT5 consensus binding-site oligonucleotide. After EGF stimulation STAT5 DNA binding activity was markedly upregulated in the nuclear extracts. The treatment of A549 cells with WT-STAT5 had no effect on STAT5 DNA binding activity (Fig. 7).

Both Stat5a $\triangle 740$ and STAT5siRNA inhibit EGF-induced STAT5 phosphorylation and DNA binding activity in A549 cells. We then examined whether Stat5a $\triangle 740$ and STAT5siRNA affected DNA-binding activity. For these studies, nuclear extracts were prepared from A549 cells infected with control adenovirus, AdStat5a $\triangle 740$, or STAT5siRNA followed by culture in the presence of $10 \%$ FBS, or during serum-free conditions in the absence or presence of EGF. We found that Stat5a $\triangle 740$ completely inhibited EGF-induced STAT5 phosphorylation (Fig. 5B). The inhibition of EGF-induced 
A

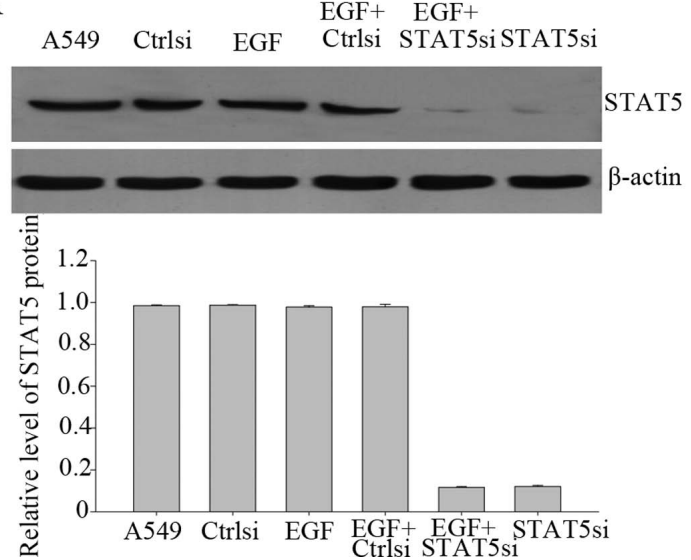

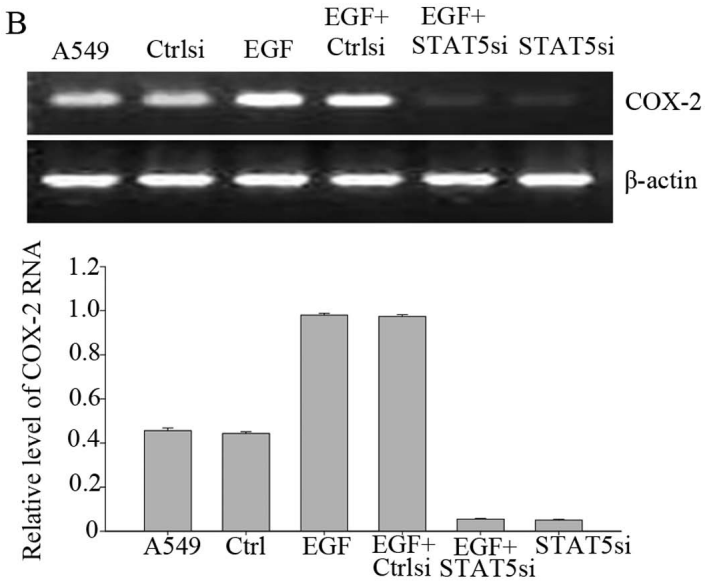

Figure 3. STAT5 siRNA inhibits both resting and EGF-induced COX-2 expression. (A) Upper panel, Western blot analysis of STAT5 expression in A549 cells transfected with STAT5siRNA together with or without EGF stimulation. A549, untransfected; Ctrlsi, transfected with control siRNA; EGF, stimulation with EGF; STAT5si, transfected with STAT5siRNA. Lower panel, quantification of STAT5 protein levels. (B) STAT5siRNA inhibited the COX-2 expression of both resting A549 cells and EGF-treated A549 cells. A549, untransfected; Ctrlsi, transfected with control siRNA; EGF, stimulation with EGF; STAT5si, transfected with STAT5siRNA. Lower panel, quantification of COX-2 RNA levels.

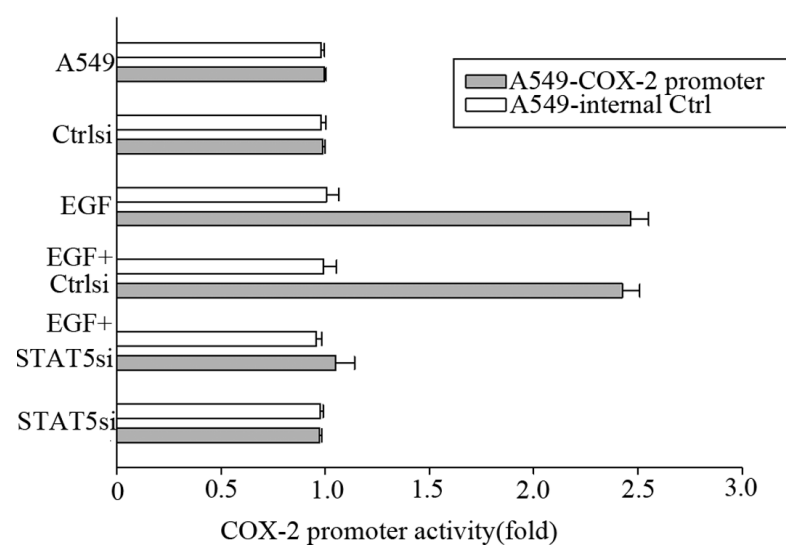

Figure 4. STAT5 siRNA inhibits COX-2 promoter transcriptional activity. A549 cells were transiently transfected with STAT5siRNA or control siRNA together with the COX-2 promoter luciferase reporter plasmid and an interna control plasmid. The transfected cells were serum-starved overnight and treated with EGF for $1 \mathrm{~h}$. Cell extracts were prepared to measure COX-2 promoter activity. A549, untransfected; Ctrlsi, transfected with control siRNA EGF, stimulation with EGF; STAT5si, transfected with STAT5siRNA. Data are expressed as the means \pm SEM (percentage of relative firefly luciferase activity, normalized to the control group without EGF stimulation, which was normalized to 1) and are representative of six independent groups.

STAT5 phosphorylation by Stat5a $\triangle 740$ was mirrored by an inhibition of DNA binding activity as measured by DNA binding assay (Fig. 7). The inhibition of phosphorylation and DNA binding was observed in A549 cells using Stat5a $\triangle 740$, whereas no effect was observed using the control adenoviral vector (control). As another control, we also tested the ability of STAT5siRNA to inhibit STAT5 activation induced by EGF. We also found that STAT5siRNA effectively inhibited EGFinduced STAT5 phosphorylation and STAT5 DNA binding activity as measured by DNA binding assay (Fig. 7).

Stat $5 a \triangle 740$ inhibits EGF-induced COX-2 expression and blocks transcriptional activity of COX-2 in A549 cells. We investigated whether there was a corresponding regulation of COX-2 by EGF and whether this required STAT5. The infection of A549 cells with Stat5a $\triangle 740$ diminished EGFinduced COX-2 expression, whereas there was no effect on the control (Fig. 5C). These data indicate that even though COX-2 can be induced by EGF, there is a still a requirement for STAT5 activity for its expression.

We then examined whether Stat $5 \mathrm{a} \triangle 740$ affected the transcriptional activity of COX-2. The COX-2 transcription was compared in the absence and presence of Stat $5 \mathrm{a} \triangle 740$ with or without EGF stimulation. When Stat5a $\triangle 740$ was transfected, stimulation with EGF had little effect on COX2-luciferase reporter gene transcription, while stimulation of EGF alone induced reporter gene transcription by approximately 2.5 -fold (Fig. 6). In contrast, WT-STAT5 did not markedly affect COX-2 activity (Fig. 6). Based on these findings, we can conclude that Stat5a $\triangle 740$ completely suppresses the transcriptional activity of COX-2 in A549 cells.

Collectively, we can conclude that the overexpression of Stat5a $\triangle 740$ effectively blocks the transcriptional activity of COX-2 in A549 cells. In contrast, WT-STAT5 does not systematically affect COX-2 promoter activity in A549 cells.

\section{Discussion}

This is the first study, to the best of our knowledge, to show that STAT5 activation mediates COX-2 expression by EGF in the A549 cell line. The main findings from our study show that: i) STAT5 is not activated in A549 cells in vitro, ii) EGF stimulation significantly increases the level of p-STAT5 protein and induces the accumulation of p-STAT5 in the nuclei, iii) STAT5 activation is crucial for COX-2 expression induced by EGF, iv) STAT5 is required for COX-2 expression but can mediate its effects through pathways that are independent of phosphorylation and transcriptional activation. We therefore propose that transcription factor STAT5 could be a potential therapeutic target for lung adenocarcinoma.

Since their discovery as key mediators of cytokine signaling, considerable progress has been made in defining the structure- 
A

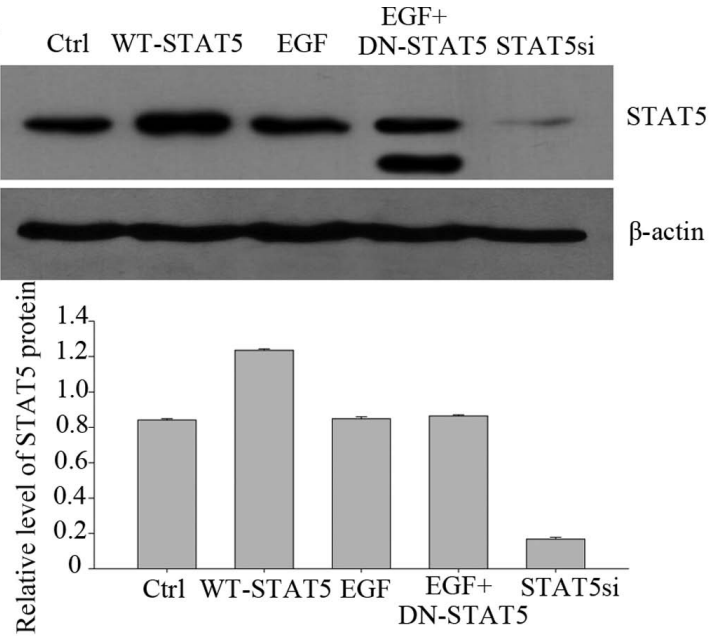

$\mathrm{C}$
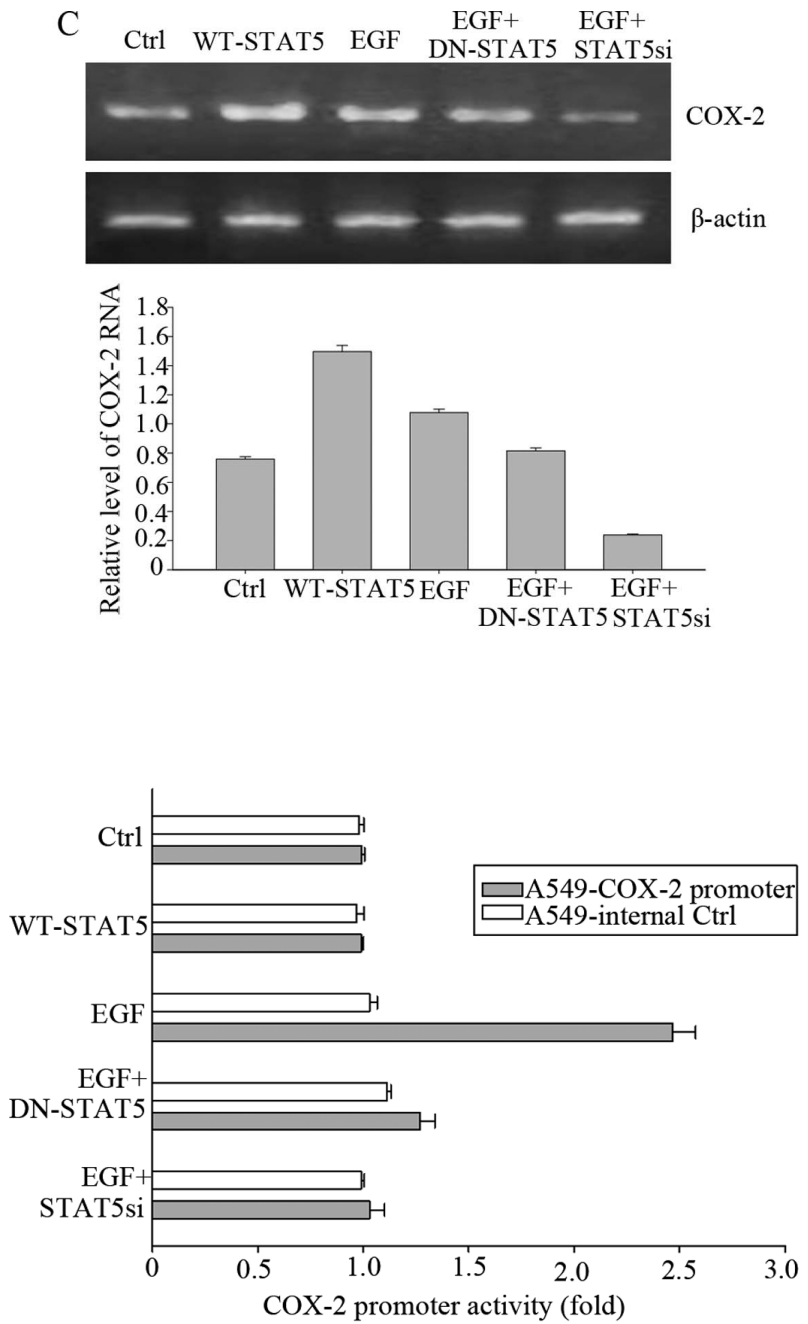

Figure 6. Stat5a $\triangle 740$ inhibits promoter transcriptional activity of COX-2 in A549 cells. A549 cells were transiently transfected with WT-STAT5, DN-STAT5 and STAT5siRNA or control adenovirus together with the COX-2 promoter luciferase reporter plasmid and an internal control plasmid. The transfected cells were serum-starved overnight and treated with EGF for $1 \mathrm{~h}$. Cell extracts were prepared to measure COX-2 promoter activity. Ctrl, transfected with control adenovirus; WT-STAT5, transfected with wild-type STAT5; EGF, stimulation with EGF; DN-STAT5, transfected with dominant-negative STAT5; STAT5si, transfected with STAT5siRNA. Data are expressed as the means \pm SEM (percentage of relative firefly luciferase activity, normalized to the control group without EGF stimulation, which was normalized to 1) and are representative of five independent groups.
B

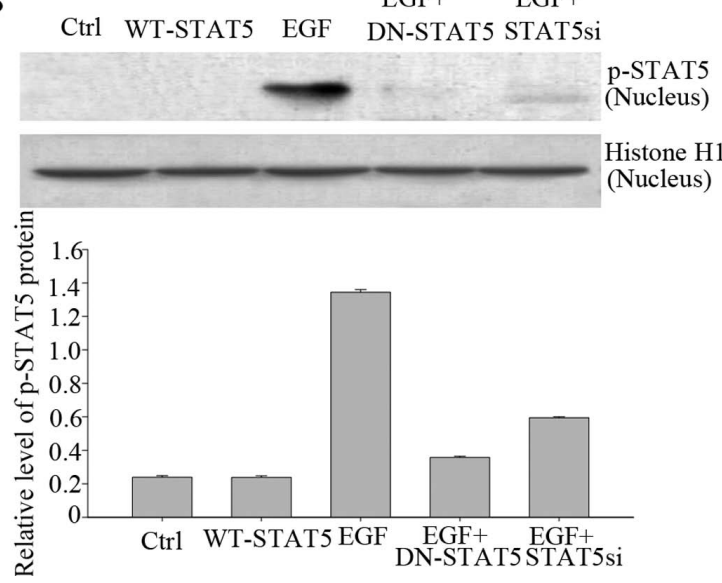

Figure 5. Both Stat5a $\triangle 740$ and STAT5siRNA inhibit EGF-induced STAT5 phosphorylation and COX-2 expression. (A) Upper panel, Western blot analysis of STAT5 expression in A549 cells transfected with WT-STAT5, DN-STAT5 and STAT5siRNA together with or without EGF stimulation. Ctrl, transfected with control adenovirus; WT-STAT5, transfected with wild-type STAT5; EGF, stimulation with EGF; DN-STAT5, transfected with dominantnegative STAT5; STAT5si, transfected with STAT5siRNA. Lower panel, quantification of STAT5 protein levels. (B) Upper panel, Western blot analysis of p-STAT5 expression in A549 cells nuclei transfected with WT-STAT5, DN-STAT5 and STAT5 siRNA with or without EGF stimulation. Ctrl, transfected with control adenovirus; WT-STAT5, transfected with wild-type STAT5; EGF, stimulation with EGF; DN-STAT5, transfected with dominantnegative STAT5; STAT5si, transfected with STAT5siRNA. Lower panel, quantification of p-STAT5 protein levels. (C) DN-STAT5 and STAT5siRNA inhibited the COX-2 expression of EGF-treated A549 cells. Otherwise, WT-STAT5 treatment increased COX-2 expression. Ctrl, transfected with control adenovirus; WT-STAT5, transfected with wild-type STAT5; EGF, stimulation with EGF; DN-STAT5, transfected with dominant-negative STAT5; STAT5si, transfected with STAT5siRNA. Lower panel, quantification of COX-2 RNA levels.

function relationships of STATs (26). In addition to their central role in normal cell signaling, many studies have demonstrated that constitutively activated STAT signaling directly contributes to oncogenesis and angiogenesis $(27,28)$. Extensive studies of primary tumors and tumor-derived cell lines have revealed that inappropriate activation of specific STATs occurs with high frequency in a wide variety of human cancers, including leukemia (29), breast cancer (24), prostate cancer (30), human non-small cell carcinoma $(31,32)$, and lung cancer (33).

The activation of STATs is ligand-, receptor- and cell typespecific and can also depend on activating stimuli within the tumor microenvironment. In general, the transcriptional activity of STATs involves their dimerization, nuclear translocation, DNA binding and recruitment of transcriptional co-activators $(34,35)$. The tyrosine phosphorylation of STATs has been shown to be essential for NSCLC (36).

STAT5 is one of the seven members of the STAT gene family of transcription factors. STAT5 is a latent cytoplasmic protein that acts both as a cytoplasmic signaling protein and a nuclear transcription factor. Phosphorylation of a specific tyrosine residue in the $\mathrm{COOH}$-terminal domain by a tyrosine kinase (37), typically of the Janus-activated kinase protein family, activates STAT5 $(38,39)$. After phosphorylation, STAT5 homodimerizes or heterodimerizes and translocates to the 


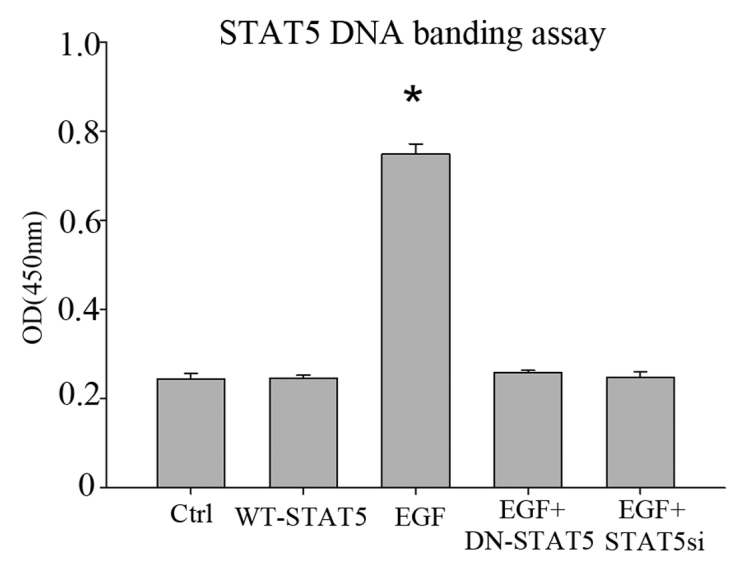

Figure 7. STAT5 DNA binding is modulated by EGF, DN-STAT5 and STAT5siRNA. Ctrl, transfected with control adenovirus; WT-STAT5, transfected with wild-type STAT5; EGF, stimulation with EGF; DN-STAT5, transfected with dominant-negative STAT5; STAT5si, transfected with STAT5siRNA. Data are the means \pm SEM. ${ }^{\text {"P }}<0.05$ when compared to the other four groups.

nucleus where it binds to specific STAT5 response elements of target gene promoters (37). In this study, we show that EGF induces STAT5 phosphorylation and we elucidate the role of EGF in the phosphorylated STAT5-mediated regulation of COX-2 transcription in A549 cells. Uniquely, our results show that the COX-2 expression is both dependent and independent of phosphorylated STAT5 in A549 cells.

In lung adenocarcinoma A549 cells, STAT5 signaling may be induced by EGF and mediated COX-2 expression. Immunofluorescence experiments with STAT5 indicated that EGF induced STAT5 phosphorylation and nuclear translocation in A549 cells. As shown by immunoblot analysis, p-STAT5 is expressed in A549 cell nuclear lysates only after treatment with EGF. This raises the possibility that STAT5 phosphorylates when exogenous EGF is present. It has been demonstrated that STAT5 is activated through EGFR family kinases $(21,40)$, which corresponds to our data of STAT5 activation through the EGFR signaling pathway in lung adenocarcinoma.

To date, STAT5 activation has been proved to be induced by IL-2, IL-3, IL-5, IL-7,IL-15, G-CSF, M-CSF, GM-CSF, erythropoietin, thrombopoietin, growth hormone, epidermal growth factor, platelet-derived growth factor and prolactin (41-48). In the present study, we demonstrate that treatment with EGF induces STAT5 activation in A549 cells. This indicates that some cytokines and growth factors can contribute to p-STAT5 expression, and that COX-2 expression is induced by endogenous cytokines in NSCLC in vivo. It has been shown that STAT5 RNA interference influences the expression level of COX-2 induced by EGF using a transient transfection assay in A549 cells. These results suggest that p-STAT5 plays a certain role, pertaining to the expression of COX-2 in activated lung adenocarcinoma. Our STAT5 RNA interference data also show that COX-2 expression is dependent on the non-phosphorylation of STAT5 in resting A549 cells, which was further confirmed by WT-STAT5 transfection.

Dominant-negative mutants are useful for analyzing gene function in human cancer cells. A common feature of dominant-negative STAT5 is the partial or complete loss of

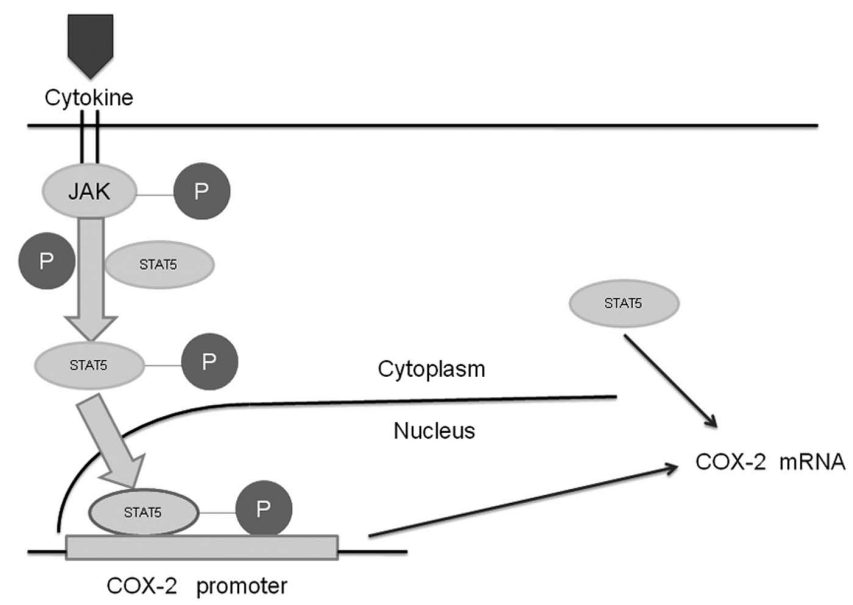

Signaling pathways of STAT5 regulating COX-2 in A549 cells

Figure 8. Schematic depiction of two different potential mechanisms of regulation: i) STAT5 is phosphorylated and translocated into the nucleus where it binds to DNA and regulates the transcription of COX-2 (phosphorylation-dependent, DNA binding-dependent), ii) non-phosphorylated STAT5 regulates COX-2 expression without phosphorylation and DNA binding (phosphorylation-independent, DNA binding-independent).

the C-terminal transactivation domain. It has been shown that Stat5a $\triangle 740$ (49), which displays a dominant-negative effect on both Stat5a and Stat5b-mediated transcription, retains the conserved tyrosine residue needed for dimerization and DNA binding. To select the dominant-negative STAT5 variant for incorporation into human lung adenocarcinoma cells, we first assessed the dominant-negative effect of STAT5 variants in A549 cells. Mutant Stat $5 \mathrm{a} \triangle 740$ was based on a naturally occurring alternative splice variant $(49,50)$. Specifically, mutant Stat5a $\triangle 740$ lacks residue Ser779, a phosphorylation site that is unique to STAT5a, but retains Ser725, a second phosphorylation site that is also present in STAT5b (51-53). Stat5a $\triangle 740$ retains residue Tyr694, the phosphorylation site that is required for dimerization and DNA binding. Our analysis showed that Stat5a $\triangle 740$ displayed dominant-negative effects against STAT5, and displayed diminished DNA binding activities. Furthermore, the loss of structural elements within the C-terminal region of STAT5 that control signal decay rate may significantly contribute to the dominant-negative properties of this type of STAT5 variants, by combining the inability to transaction with increased DNA binding activity. In this study, we provide evidence that dominant-negative STAT5 ablates the EGFinduced stimulation of DNA binding activity (Fig. 7). Stat5a $\triangle 740$ effectively inhibited EGF-induced STAT5 phosphorylation and blocked the transcriptional activity of COX-2 in A549 cells.

We have shown that STAT5 mediates and regulates the activation of COX-2 by EGF stimulation in A549 cells, suggesting a novel biological role for STAT5 in the regulation of COX-2 signaling pathways. A previous report demonstrated that treatment with EGF $(20 \mathrm{ng} / \mathrm{ml})$ did not induce COX-2 expression in A549 cells compared to the control in serumstarved or serum-supplemented conditions at any of the time-points studied (1, 2, 8 and $24 \mathrm{~h})$ (54). Stimulation of A549 cells with EGF at a concentration of $20 \mathrm{ng} / \mathrm{ml}$, was probably not enough to induce COX-2 expression. Moreover, 
there was no internal control in this study. We have shown in the present study that EGF $(100 \mathrm{ng} / \mathrm{ml})$ increases the expression levels of COX-2 RNA in lung adenocarcinoma cells (Fig. 3B). STAT5 may contribute to the COX-2 upregulation directly through STAT5 binding sites in the COX-2 promoter. Our data showing that the activity of a COX-2 promoter-luciferase reporter construct is enhanced by EGF stimulation in A549 cells provides additional evidence that EGF can modulate the transcriptional activity of COX-2 (Fig. 4). This is an important step in understanding the latent response to EGF during lung cancer development (tumorigenesis), as well as a potential role in lung cancer.

In conclusion, STAT5 activation induced by EGF increases COX-2 expression, which can be mediated by EGFR activation in lung adenocarcinoma cells. This study also shows that non-phosphorylated STAT5 can directly influence COX-2 expression in resting A549 cells, suggesting the requirement of a yet undefined intermediate signaling pathway. Finally, our findings show that COX-2 expression is dependent on STAT5 phosphorylation, and that a second pathway exists independent of EGF that does not require STAT5 phosphorylation (Fig. 8). New therapies targeting COX-2-dependent genes, such as STAT5, could be beneficial in the treatment of lung cancer.

\section{Acknowledgments}

This study was supported by the Chinese National Natural Science Foundation (grant no. 30300100). We thank Professor Anhua Wu and Baozhong Shen for their thoughtful comments. We are grateful to Dr Xiaofei Qin for providing WT-STAT5 and DN-STAT5 expression plasmids.

\section{References}

1. Alroy I and Yarden Y: The ErbB signaling network in embryogenesis and oncogenesis: signal diversification through combinatorial ligand-receptor interactions. FEBS Lett 410: 83-86, 1997.

2. Riese DJ II and Stern DF: Specificity within the EGF family/ErbB receptor family signaling network. Bioessays 20: 41-48, 1998.

3. De Luca A, Carotenuto A, Rachiglio A, et al: The role of the EGFR signaling in tumor microenvironment. J Cell Physiol 214: 559-567, 2008

4. Bonanno L, Schiavon M, Nardo G, et al: Prognostic and predictive implications of EGFR mutations, EGFR copy number and KRAS mutations in advanced stage lung adenocarcinoma. Anticancer Res 30: 5121-5128, 2010.

5. Gu L, Vogiatzi P, Puhr M, et al: Stat5 promotes metastatic behavior of human prostate cancer cells in vitro and in vivo. Endocr Relat Cancer 17: 481-493, 2010.

6. Sung YH, Park J, Choi B, et al: Hematopoietic malignancies associated with increased Stat5 and $\mathrm{Bcl}-\mathrm{x}(\mathrm{L})$ expressions in Ink4a/Arf-deficient mice. Mech Ageing Dev 126: 732-739, 2005.

7. Wagner KU and Rui H: Jak2/Stat5 signaling in mammogenesis, breast cancer initiation and progression. J Mammary Gland Biol Neoplasia 13: 93-103, 2008.

8. Smith WL, DeWitt DL and Garavito RM: Cyclooxygenases: structural, cellular, and molecular biology. Annu Rev Biochem 69: $145-182,2000$

9. Morita I: Distinct functions of COX-1 and COX-2. Prostaglandins Other Lipid Mediat 68-69: 165-175, 2002.

10. Takeuchi K, Tanaka A, Suzuki K and Mizoguchi H: Gastrointestinal sparing anti-inflammatory drugs - effects on ulcerogenic and healing responses. Curr Pharm Des 7: 49-69, 2001.

11. Schwab JM, Schluesener HJ, Meyermann R and Serhan CN: COX-3 the enzyme and the concept: steps towards highly specialized pathways and precision therapeutics? Prostaglandins Leukot Essent Fatty Acids 69: 339-343, 2003.
12. Husvik $C$, Bryne $M$ and Halstensen TS: Epidermal growth factor-induced cyclooxygenase-2 expression in oral squamous cell carcinoma cell lines is mediated through extracellular signalregulated kinase 1/2 and p38 but is Src and nuclear factor-kappa B independent. Eur J Oral Sci 117: 528-535, 2009.

13. Seo M and Juhnn YS: Gq protein mediates UVB-induced cyclooxygenase-2 expression by stimulating HB-EGF secretion from HaCaT human keratinocytes. Biochem Biophys Res Commun 393: 190-195, 2010.

14. Rasanen K and Vaheri A: TGF-betal causes epithelial-mesenchymal transition in $\mathrm{HaCaT}$ derivatives, but induces expression of COX-2 and migration only in benign, not in malignant keratinocytes. J Dermatol Sci 58: 97-104, 2010.

15. Bage T, Lindberg J, Lundeberg J, Modeer T and Yucel-Lindberg T: Signal pathways JNK and NF-kappaB, identified by global gene expression profiling, are involved in regulation of TNFalphainduced mPGES-1 and COX-2 expression in gingival fibroblasts. BMC Genomics 11: 241, 2010.

16. Bradbury DA, Newton R, Zhu YM, et al: Effect of bradykinin, TGF-beta1, IL-1beta, and hypoxia on COX-2 expression in pulmonary artery smooth muscle cells. Am J Physiol Lung Cell Mol Physiol 283: L717-L725, 2002.

17. Chan E, Lafleur B, Rothenberg ML, et al: Dual Blockade of the EGFR and COX-2 Pathways: A Phase II Trial of Cetuximab and Celecoxib in Patients With Chemotherapy Refractory Metastatic Colorectal Cancer. Am J Clin Oncol: Jan 6, 2011 (Epub ahead of print).

18. Liu X, Li P, Zhang ST, You H, Jia JD and Yu ZL: COX-2 mRNA expression in esophageal squamous cell carcinoma (ESCC) and effect by NSAID. Dis Esophagus 21: 9-14, 2008.

19. Blanco A, Habib A, Levy-Toledano S and Maclouf J: Involvement of tyrosine kinases in the induction of cyclo-oxygenase- 2 in human endothelial cells. Biochem J 312 (Pt 2): 419-423, 1995.

20. Allport VC, Slater DM, Newton R and Bennett PR: NF-kappaB and AP-1 are required for cyclo-oxygenase 2 gene expression in amnion epithelial cell line (WISH). Mol Hum Reprod 6: $561-565,2000$.

21. Chen LC, Chen BK and Chang WC: Activating protein 1mediated cyclooxygenase-2 expression is independent of $\mathrm{N}$-terminal phosphorylation of c-Jun. Mol Pharmacol 67: 2057-2069, 2005.

22. Fan XM, Wong BC, Lin MC, et al: Interleukin-1beta induces cyclo-oxygenase- 2 expression in gastric cancer cells by the p38 and p44/42 mitogen-activated protein kinase signaling pathways. J Gastroenterol Hepatol 16: 1098-1104, 2001.

23. Jung YJ, Isaacs JS, Lee S, Trepel J and Neckers L: IL-1betamediated up-regulation of HIF-1alpha via an NFkappaB/COX-2 pathway identifies HIF-1 as a critical link between inflammation and oncogenesis. FASEB J 17: 2115-2117, 2003.

24. Yamashita H, Iwase H, Toyama T and Fujii Y: Naturally occurring dominant-negative Stat5 suppresses transcriptional activity of estrogen receptors and induces apoptosis in T47D breast cancer cells. Oncogene 22: 1638-1652, 2003.

25. Wu A, Ericson K, Chao W and Low WC: NFAT and AP1 are essential for the expression of a glioblastoma multiforme related IL-13Ra2 transcript. Cell Oncol 32: 313-329, 2010.

26. Darnell JE Jr: STATs and gene regulation. Science 277: 1630-1635, 1997.

27. Bowman T, Garcia R, Turkson J and Jove R: STATs in oncogenesis. Oncogene 19: 2474-2488, 2000.

28. Niu G, Wright KL, Huang M, et al: Constitutive Stat3 activity up-regulates VEGF expression and tumor angiogenesis. Oncogene 21: 2000-2008, 2002.

29. Lin TS, Mahajan S and Frank DA: STAT signaling in the pathogenesis and treatment of leukemias. Oncogene 19: 2496-2504, 2000 .

30. Li H, Ahonen TJ, Alanen K, et al: Activation of signal transducer and activator of transcription 5 in human prostate cancer is associated with high histological grade. Cancer Res 64: 4774-4782, 2004.

31. Grandis JR, Drenning SD, Zeng Q, et al: Constitutive activation of Stat 3 signaling abrogates apoptosis in squamous cell carcinogenesis in vivo. Proc Natl Acad Sci USA 97: 4227-4232, 2000.

32. Xi S, Zhang Q, Dyer KF, et al: Src kinases mediate STAT growth pathways in squamous cell carcinoma of the head and neck. J Biol Chem 278: 31574-31583, 2003.

33. Song L, Turkson J, Karras JG, Jove R and Haura EB: Activation of Stat 3 by receptor tyrosine kinases and cytokines regulates survival in human non-small cell carcinoma cells. Oncogene 22: 4150-4165, 2003. 
34. Klampfer L: Signal transducers and activators of transcription (STATs): Novel targets of chemopreventive and chemotherapeutic drugs. Curr Cancer Drug Targets 6: 107-121, 2006.

35. Vinkemeier U: Getting the message across, STAT! Design principles of a molecular signaling circuit. J Cell Biol 167: 197-201, 2004

36. Chang KT, Tsai CM, Chiou YC, Chiu CH, Jeng KS and Huang CY: IL- 6 induces neuroendocrine dedifferentiation and cell proliferation in non-small cell lung cancer cells. Am J Physiol Lung Cell Mol Physiol 289: L446-L453, 2005.

37. Ihle JN: The Stat family in cytokine signaling. Curr Opin Cell Biol 13: 211-217, 2001

38. Rui H, Djeu JY, Evans GA, Kelly PA and Farrar WL: Prolactin receptor triggering. Evidence for rapid tyrosine kinase activation. J Biol Chem 267: 24076-24081, 1992.

39. Rui H, Lebrun JJ, Kirken RA, Kelly PA and Farrar WL: JAK2 activation and cell proliferation induced by antibody-mediated prolactin receptor dimerization. Endocrinology 135: 1299-1306, 1994.

40. Mirmohammadsadegh A, Hassan M, Bardenheuer W, et al: STAT5 phosphorylation in malignant melanoma is important for survival and is mediated through SRC and JAK1 kinases. J Invest Dermatol 126: 2272-2280, 2006.

41. Mui AL, Wakao H, O'Farrell AM, Harada N and Miyajima A: Interleukin-3, granulocyte-macrophage colony stimulating factor and interleukin-5 transduce signals through two STAT5 homologs. EMBO J 14: 1166-1175, 1995.

42. Lai CF, Ripperger J, Morella KK, et al: STAT3 and STAT5B are targets of two different signal pathways activated by hematopoietin receptors and control transcription via separate cytokine response elements. J Biol Chem 270: 23254-23257, 1995.

43. Novak U, Mui A, Miyajima A and Paradiso L: Formation of STAT5-containing DNA binding complexes in response to colony-stimulating factor-1 and platelet-derived growth factor. J Biol Chem 271: 18350-18354, 1996.

44. Wakao H, Harada N, Kitamura T, Mui AL and Miyajima A: Interleukin 2 and erythropoietin activate STAT5/MGF via distinct pathways. EMBO J 14: 2527-2535, 1995.
45. Gouilleux F, Pallard C, Dusanter-Fourt I, et al: Prolactin, growth hormone, erythropoietin and granulocyte-macrophage colony stimulating factor induce MGF-Stat5 DNA binding activity. EMBO J 14: 2005-2013, 1995.

46. Pallard C, Gouilleux F, Benit L, et al: Thrombopoietin activates a STAT5-like factor in hematopoietic cells. EMBO J 14: 2847-2856, 1995.

47. Ruff-Jamison S, Chen K and Cohen S: Epidermal growth factor induces the tyrosine phosphorylation and nuclear translocation of Stat 5 in mouse liver. Proc Natl Acad Sci USA 92: 4215-4218, 1995.

48. Gouilleux F, Wakao H, Mundt M and Groner B: Prolactin induces phosphorylation of Tyr694 of Stat5 (MGF), a prerequisite for DNA binding and induction of transcription. EMBO J 13: 4361-4369, 1994

49. Kazansky AV, Raught B, Lindsey SM, Wang YF and Rosen JM: Regulation of mammary gland factor/Stat5a during mammary gland development. Mol Endocrinol 9: 1598-1609, 1995.

50. Wang D, Stravopodis D, Teglund S, Kitazawa J and Ihle JN: Naturally occurring dominant negative variants of Stat5. Mol Cell Biol 16: 6141-6148, 1996.

51. Yamashita H, Nevalainen MT, Xu J, et al: Role of serine phosphorylation of Stat5a in prolactin-stimulated beta-casein gene expression. Mol Cell Endocrinol 183: 151-163, 2001.

52. Beuvink I, Hess D, Flotow H, Hofsteenge J, Groner B and Hynes NE: Stat5a serine phosphorylation. Serine 779 is constitutively phosphorylated in the mammary gland, and serine 725 phosphorylation influences prolactin-stimulated in vitro DNA binding activity. J Biol Chem 275: 10247-10255, 2000.

53. Yamashita H, Xu J, Erwin RA, Farrar WL, Kirken RA and Rui $H$ Differential control of the phosphorylation state of prolinejuxtaposed serine residues Ser725 of Stat5a and Ser730 of Stat5b in prolactin-sensitive cells. J Biol Chem 273: 30218-30224, 1998

54. Richardson CM, Richardson D, Swinson DE, Swain WA, Cox G and O'Byrne KJ: Cyclooxygenase-2 protein levels are independent of epidermal growth factor receptor expression or activation in operable non-small cell lung cancer. Lung Cancer 48: 47-57, 2005. 